\title{
Kolmogorov kernel estimates for the Ornstein-Uhlenbeck operator
}

\author{
Robert HaLler-DintelmanN AND JULIAN WIEDL
}

\begin{abstract}
Replacing the Gaussian semigroup in the heat kernel estimates by the Ornstein-Uhlenbeck semigroup on $\mathbb{R}^{d}$, we define the notion of Kolmogorov kernel estimates. This allows us to show that under Dirichlet boundary conditions Ornstein-Uhlenbeck operators are generators of consistent, positive, (quasi-) contractive $C_{0}$-semigroups on $L^{p}(\Omega)$ for all $1 \leq p<\infty$ and for every domain $\Omega \subseteq \mathbb{R}^{d}$. For exterior domains with sufficiently smooth boundary a result on the location of the spectrum of these operators is also given.
\end{abstract}

Mathematics Subject Classification (2000): 47D06 (primary); 35K20 (secondary).

\section{Introduction}

Heat kernel estimates have proved to be a powerful tool for the analysis of elliptic differential operators. Besides many other things, they allow the extension of a given semigroup on $L^{p_{0}}$ for some $p_{0}$ to the whole scale of $L^{p}$-spaces for $1 \leq p<$ $\infty$ in a consistent way, transferring certain nice properties to all these semigroups, such as analyticity, and yielding $p$-invariance of the spectrum.

Dealing with Ornstein-Uhlenbeck operators on $L^{p}(\Omega)$ for unbounded domains $\Omega$ we evidently cannot expect to get heat kernel estimates, as the spectrum of these operators in $L^{p}\left(\mathbb{R}^{d}\right)$ already depends heavily on $p$. Nevertheless, the well-known representation for the Ornstein-Uhlenbeck semigroup on $L^{p}\left(\mathbb{R}^{d}\right)$ that is due to A. N. Kolmogorov and given by

$$
T(t) f(x)=\int_{\mathbb{R}^{d}} k_{t}\left(\mathrm{e}^{t B} x-y\right) f(y) \mathrm{d} y=\left(k_{t} * f\right)\left(\mathrm{e}^{t B} x\right),
$$

where the Kolmogorov kernel is

$$
k_{t}(x)=\frac{1}{(4 \pi)^{\frac{d}{2}}\left(\operatorname{det} Q_{t}\right)^{\frac{1}{2}}} \exp \left(-\frac{1}{4} Q_{t}^{-1} x \cdot x\right) \quad \text { with } \quad Q_{t}=\int_{0}^{t} \mathrm{e}^{s B} Q \mathrm{e}^{s B^{\star}} \mathrm{d} s,
$$

Pervenuto alla Redazione il 4 luglio 2005 e in forma definitiva il 7 novembre 2005. 
looks very similar to the heat semigroup. So it is a natural idea to consider Kolmogorov kernel estimates replacing the Gaussian semigroup by the Kolmogorov semigroup $(T(t))_{t \geq 0}$, thus getting a majorising semigroup that is well adapted to operators of the Ornstein-Uhlenbeck type. These estimates then allow us to extend semigroups on $L^{p_{0}}$ to the whole scale of $L^{p}$-spaces for $1 \leq p<\infty$, analogously to the case of heat kernel bounds.

Having established this idea, in the sequel we apply it to Ornstein-Uhlenbeck operators with Dirichlet boundary conditions in $L^{p}(\Omega)$, where $1 \leq p<\infty$ and $\Omega$ is a domain in $\mathbb{R}^{d}$. Ornstein-Uhlenbeck operators are differential operators, formally given by

$$
(\mathcal{A} u)(x)=\sum_{i, j=1}^{d} q_{i j} D_{i} D_{j} u(x)+B x \cdot \nabla u(x),
$$

where $Q=\left(q_{i j}\right)_{i, j=1}^{d} \in \mathbb{R}^{d \times d}$ is a symmetric and positive definite matrix and $B=\left(b_{i j}\right)_{i, j=1}^{d} \in \mathbb{R}^{d \times d} \backslash\{0\}$. They first appeared in stochastic analysis, describing a Brownian motion with an additional drift. In this context one usually works in spaces of continuous functions on $\mathbb{R}^{d}$ or in the spaces $L^{p}\left(\mathbb{R}^{d}, \mu\right)$, where $\mu$ is the invariant measure of the underlying process.

Recently, it became clear that an analytic treatment of these operators is of great interest. For instance, looking at the Stokes equation in the exterior of a rotating obstacle leads to operators of the Ornstein-Uhlenbeck type, see [14], [13] and [9]. Thus one is interested in their behaviour on $L^{p}(\Omega)$ with respect to the Lebesgue measure for domains and especially exterior domains.

Passing from the invariant measure to the Lebesgue measure changes the properties of the operator completely. The spectrum is no longer contained in the negative real axis, instead it contains a vertical line (cf. [15]), so the semigroup $(T(t))_{t \geq 0}$ on $L^{p}\left(\mathbb{R}^{d}\right)$ is not analytic, as it is in $L^{p}\left(\mathbb{R}^{d}, \mu\right)$ (cf. [16]), and it even fails to be eventually norm-continuous.

Whereas Ornstein-Uhlenbeck operators are well understood in $L^{p}\left(\mathbb{R}^{d}\right)$ (cf. $[15,18,17])$ and for bounded domains, where they can be viewed as a perturbation of lower order of the elliptic diffusion part, there are very few results for unbounded domains. M. Geissert, H. Heck, M. Hieber and I. Wood showed in [10] that in the case of an exterior domain $\Omega$ with sufficiently smooth boundary, a realisation of $\mathcal{A}$ on $L^{p}(\Omega)$ generates a $C_{0}$-semigroup and G. da Prato and A. Lunardi treat the case of $L^{2}$-spaces of convex sets with respect to Neumann boundary conditions and infinitesimally invariant measures in [4]. The Dirichlet problem in spaces of bounded continuous functions on smooth domains is treated by S. Fornaro, G. Metafune and E. Priola in [8]. An overview may be found in [3].

In this paper we show that for arbitrary domains $\Omega \subseteq \mathbb{R}^{d}$ a realisation of $\mathcal{A}$ in $L^{2}(\Omega)$ generates a (quasi-)contractive, positive $C_{0}$-semigroup, that has a Kolmogorov kernel estimate, see Theorem 3.2. In the sequel this allows us to define consistent Ornstein-Uhlenbeck semigroups on $L^{p}(\Omega)$ for $1 \leq p<\infty$ that have the same contractivity, positivity and domination properties. This immediately gives 
an upper bound on the growth bound of the semigroups and implies that OrnsteinUhlenbeck operators admit a bounded $H^{\infty}$-calculus on $L^{p}(\Omega)$ (Theorem 4.5).

In the special case of an exterior domain with sufficiently smooth boundary, it turns out that the domain is

$$
W_{0}^{1, p}(\Omega) \cap W^{2, p}(\Omega) \cap\left\{f \in L^{p}(\Omega): B x \cdot \nabla f \in L^{p}(\Omega)\right\}
$$

and we even deduce in Theorem 5.2 that the same vertical line as in the case of the whole space is contained in the spectrum of the operator, so the spectral behaviour is the same as for the case $\Omega=\mathbb{R}^{d}$. This means that also in this case the semigroup is not eventually norm-continuous. Nevertheless we can show that its growth bound and the spectral bound of its generator coincide, which is no longer clear by standard spectral theory for semigroups.

The paper is organised as follows. In section 2 we introduce the notion of Kolmogorov kernel estimates and prove their main implications. The generation result for $\mathcal{A}$ in $L^{2}(\Omega)$ is contained in section 3 and in section 4 we show that this semigroup is positive and admits a Kolmogorov kernel estimate. Section 5 finally contains the results for exterior domains.

Notation. Throughout this paper we use the following notation.

For a closed operator $(A, D(A))$ on some Banach space $X$ we denote by $\sigma(A)$ the spectrum, by $\varrho(A)$ the resolvent set and by $R(\lambda, A)=(\lambda-A)^{-1}, \lambda \in \varrho(A)$, the resolvent of $A$. Furthermore, the space of all bounded linear operators on $X$ is denoted by $\mathcal{L}(X)$.

As usual, for $\Omega \subseteq \mathbb{R}^{d}$ open, $\|\cdot\|_{p}$ stands for the norm of the Lebesgue spaces $L^{p}(\Omega)$ whenever the set $\Omega$ is clear from the context. We write $W^{k, p}(\Omega)$, or $H^{k}(\Omega)$ in the case $p=2$, for the Sobolev spaces, $C_{c}^{\infty}(\Omega)$ for the space of all smooth functions having compact support in $\Omega$ and $W_{0}^{1, p}(\Omega)$, or $H_{0}^{1}(\Omega)$, is the closure of $C_{c}^{\infty}(\Omega)$ in the norm of $W^{1, p}(\Omega)$ or $H^{1}(\Omega)$, respectively. Furthermore, if $X$ is a function space, $X_{+}$stands for the cone of all positive functions in $X$.

Finally, $B_{r}\left(x_{0}\right)$ is the open ball of radius $r$ with centre $x_{0}$ and, given a matrix $B \in \mathbb{R}^{d \times d}$, we write $\operatorname{tr}(B)=\sum_{j=1}^{d} b_{j j}$ for its trace.

\section{Kolmogorov kernel estimates}

Given a matrix $B \in \mathbb{R}^{d \times d} \backslash\{0\}$ and a positive definite matrix $Q \in \mathbb{R}^{d \times d}$, we define the Kolmogorov semigroup $\left(K_{p}(t)\right)_{t \geq 0}$, on $L^{p}\left(\mathbb{R}^{d}\right)$ by

$$
\left(K_{p}(t) f\right)(x)=\int_{\mathbb{R}^{d}} k_{t}\left(\mathrm{e}^{t B} x-y\right) f(y) \mathrm{d} y, \quad f \in L^{p}\left(\mathbb{R}^{d}\right),
$$

where the Kolmogorov kernel $k_{t}$ is given by

$$
k_{t}(x)=\frac{1}{(4 \pi)^{\frac{d}{2}}\left(\operatorname{det} Q_{t}\right)^{\frac{1}{2}}} \exp \left(-\frac{1}{4} Q_{t}^{-1} x \cdot x\right) \quad \text { with } \quad Q_{t}=\int_{0}^{t} \mathrm{e}^{s B} Q \mathrm{e}^{s B^{\star}} \mathrm{d} s .
$$


It is well known (cf. [15]) that $\left(K_{p}(t)\right)_{t \geq 0}$ is a positive $C_{0}$-semigroup on $L^{p}\left(\mathbb{R}^{d}\right)$ for every $1 \leq p<\infty$ and it is straightforward by substitution and Young's inequality that for every $f \in L^{p}\left(\mathbb{R}^{d}\right)$

$$
\begin{aligned}
\left\|K_{p}(t) f\right\|_{p} & =\left(\int_{\mathbb{R}^{d}}\left|\left(k_{t} * f\right)\left(\mathrm{e}^{t B} x\right)\right|^{p} \mathrm{~d} x\right)^{\frac{1}{p}} \\
& =\mathrm{e}^{-\frac{\operatorname{tr}(B)}{p} t}\left\|k_{t} * f\right\|_{p} \leq \mathrm{e}^{-\frac{\operatorname{tr}(B)}{p} t}\|f\|_{p},
\end{aligned}
$$

since $\left\|k_{t}\right\|_{1}=1$. The generator $A_{\mathbb{R}^{d}, p}$ of this semigroup is the Ornstein-Uhlenbeck operator $\mathcal{A}$ given in (1.1) and G. Metafune, J. Prüss, A. Rhandi, and R. Schnaubelt showed in [18] that its domain is

$$
D\left(A_{\mathbb{R}^{d}, p}\right)=W^{2, p}\left(\mathbb{R}^{d}\right) \cap\left\{f \in L^{p}\left(\mathbb{R}^{d}\right): B x \cdot \nabla f \in L^{p}\left(\mathbb{R}^{d}\right)\right\}
$$

for $1<p<\infty$.

Now, let $\Omega \subseteq \mathbb{R}^{d}$ be an open set, $1 \leq p<\infty$, and let $(T(t))_{t \geq 0}$ be a $C_{0^{-}}$ semigroup on $L^{p}(\Omega)$ with generator $A$.

Definition 2.1. We say that the semigroup $(T(t))_{t \geq 0}$ on $L^{p}(\Omega)$ satisfies a Kolmogorov kernel estimate if there exist a matrix $B \in \mathbb{R}^{d \times d} \backslash\{0\}$, a positive definite matrix $Q \in \mathbb{R}^{d \times d}, M \geq 0$ and $\omega \in \mathbb{R}$, such that for all $f \in L^{p}(\Omega)$ and all $t \geq 0$ we have the pointwise estimate

$$
|T(t) f| \leq M \mathrm{e}^{\omega t} K_{p}(t)|\tilde{f}|,
$$

where $\tilde{f}$ denotes the trivial extension of $f$ to $\mathbb{R}^{d}$.

For such semigroups we have the following result.

Proposition 2.2. Let $\Omega \subseteq \mathbb{R}^{d}$ be open, $1 \leq p<\infty$, and let $(T(t))_{t \geq 0}$ be a $C_{0^{-}}$ semigroup on $L^{p}(\Omega)$ that satisfies a Kolmogorov kernel estimate for some matrix $B$, a positive definite matrix $Q$ and constants $M$ and $\omega$. Then for $1 \leq q<\infty$ there exist consistent $C_{0}$-semigroups $\left(T_{q}(t)\right)_{t \geq 0}$ on $L^{q}(\Omega)$, such that $T_{p}=T$ and

$$
\left\|T_{q}(t)\right\|_{\mathcal{L}\left(L^{q}(\Omega)\right)} \leq M \mathrm{e}^{\left(\omega-\frac{\mathrm{tr}(B)}{q}\right) t}
$$

for every $t \geq 0$. Furthermore, $T_{q}$ satisfies the same Kolmogorov kernel estimate and if $T$ is a positive semigroup, $T_{q}$ is also positive.

Proof. Let $g \in L^{p}(\Omega) \cap L^{q}(\Omega)$ and $t \geq 0$. Then, by consistency of the semigroups $K_{q}$ for $1 \leq q<\infty$ and (2.1), we have

$$
\begin{aligned}
\|T(t) g\|_{q} & =\left(\int_{\Omega}|T(t) g|^{q}\right)^{\frac{1}{q}} \leq M \mathrm{e}^{\omega t}\left(\int_{\mathbb{R}^{d}}\left(K_{p}(t)|\tilde{g}|\right)^{q}\right)^{\frac{1}{q}} \\
& =M \mathrm{e}^{\omega t}\left\|K_{q}(t)|\tilde{g}|\right\|_{q} \leq M \mathrm{e}^{\left(\omega-\frac{\mathrm{t}(B)}{q}\right) t}\|g\|_{q} .
\end{aligned}
$$


Thus we can extend the operator $T(t)$ continuously to an operator $T_{q}(t)$ on $L^{q}(\Omega)$, obtaining $C_{0}$-semigroups that are consistent by construction and that obey the stated norm estimate by the above calculation.

For $f \in L^{q}(\Omega) \cap L^{p}(\Omega)$ the inequality

$$
\left|T_{q}(t) f\right| \leq M \mathrm{e}^{\omega t} K_{q}(t)|\tilde{f}|
$$

is immediate by consistency. Thus $M \mathrm{e}^{\omega t} K_{q}(t)|\tilde{f}|-\left|T_{q}(t) f\right|$ is positive for all these functions and the Kolmogorov kernel estimates follow for arbitrary $f \in L^{q}(\Omega)$, since $L^{q}(\Omega)_{+}$is closed in $L^{q}(\Omega)$.

Finally, the same closedness argument yields the positivity of $T_{q}$, whenever $T$ is positive.

For every $1 \leq q<\infty$ we denote the generator of $\left(T_{q}(t)\right)_{t \geq 0}$ by $A_{q}$. The Kolmogorov kernel estimates also provide consistency results for these operators and their resolvents. We collect them in the next proposition.

Proposition 2.3. Let $1 \leq q<\infty$. Then

1. $R\left(\lambda, A_{q}\right) f=R\left(\lambda, A_{r}\right) f$ for all $f \in L^{q}(\Omega) \cap L^{r}(\Omega)$, all $1 \leq r<\infty$ and all $\lambda \in \mathbb{C}$ with $\operatorname{Re}(\lambda)>\lambda_{0}:=\max \left(\omega-\frac{\operatorname{tr}(B)}{q}, \omega-\frac{\operatorname{tr}(B)}{r}\right)$.

2. The set $\left\{f \in D(A) \cap L^{q}(\Omega): A f \in L^{q}(\Omega)\right\}$ is contained in $D\left(A_{q}\right)$ and $A_{q} f=$ Af for all such $f$.

3. $\left|R\left(\lambda, A_{q}\right) f\right| \leq M R\left(\lambda, \omega+A_{\mathbb{R}^{d}, q}\right)|\tilde{f}|$ for all $f \in L^{q}(\Omega)$ and all $\lambda>\omega-\frac{\operatorname{tr}(B)}{q}$. Proof.

1. By Proposition 2.2 we know that the growth bounds of $T_{q}$ and $T_{r}$ are at most $\lambda_{0}$. Taking $\lambda \in \mathbb{C}$ with $\operatorname{Re}(\lambda)>\lambda_{0}$, this allows us to conclude with the help of the Laplace transform

$$
R\left(\lambda, A_{q}\right) f=\int_{0}^{\infty} \mathrm{e}^{-\lambda t} T_{q}(t) f \mathrm{~d} t=\int_{0}^{\infty} \mathrm{e}^{-\lambda t} T_{r}(t) f \mathrm{~d} t=R\left(\lambda, A_{r}\right) f
$$

for every $f \in L^{q}(\Omega) \cap L^{r}(\Omega)$ by the consistency of the semigroups.

2. Let $f \in D(A) \cap L^{q}(\Omega)$ with $A f \in L^{q}(\Omega)$. Then, choosing $\lambda>\omega+|\operatorname{tr}(B)|$, we have $\lambda \in \varrho(A) \cap \varrho\left(A_{q}\right)$ and since $(\lambda-A) f \in L^{p}(\Omega) \cap L^{q}(\Omega)$, we get

$$
f=R(\lambda, A)(\lambda-A) f=R\left(\lambda, A_{q}\right)(\lambda-A) f \in D\left(A_{q}\right)
$$

by part (1) of this proof. The above equality also yields $A_{q} f=A f$.

3. Let $\lambda>\omega-\frac{\operatorname{tr}(B)}{q}$. Then we may again use Laplace transform for $R\left(\lambda, A_{q}\right)$ and we obtain for $f \in L^{q}(\Omega)$

$$
\begin{aligned}
\left|R\left(\lambda, A_{q}\right) f\right| & =\left|\int_{0}^{\infty} \mathrm{e}^{-\lambda t} T_{q}(t) f \mathrm{~d} t\right| \\
& \leq M \int_{0}^{\infty} \mathrm{e}^{-\lambda t} \mathrm{e}^{\omega t} K_{q}(t)|\tilde{f}| \mathrm{d} t=M R\left(\lambda, \omega+A_{\mathbb{R}^{d}, q}\right)|\tilde{f}|,
\end{aligned}
$$

since $T_{q}$ also has a Kolmogorov kernel estimate by Proposition 2.2. 
In the special case that $M=1$ in the kernel estimates, the operators $A_{q}-$ $\omega+\operatorname{tr}(B) / q$ generate contraction semigroups for all $q \in(1, \infty)$. Since $|\operatorname{tr}(B) / q| \leq$ $|\operatorname{tr}(B)|$ for all $q \in(1, \infty)$, the amount of the shift is bounded. Thus $A_{q}-\omega-|\operatorname{tr}(B)|$, $1<q<\infty$, is a family of generators of contraction semigroups on $L^{q}(\Omega)$. If in addition the semigroup $(T(t))_{t \geq 0}$ is positive, by Proposition 2.2 all the semigroups are positive. This immediately yields a bounded $H^{\infty}$-calculus for their generators, see [12].

Proposition 2.4. The operators $A_{q}-\omega-|\operatorname{tr}(B)|$ admit a bounded $H^{\infty}$-calculus for every $1<q<\infty$, whenever $(T(t))_{t \geq 0}$ is a positive semigroup satisfying $a$ Kolmogorov kernel estimate with $M=1$.

\section{The Ornstein-Uhlenbeck semigroup on $L^{2}(\Omega)$}

In the following we want to use Kolmogorov kernel estimates to show that the Ornstein-Uhlenbeck operator $\mathcal{A}$ is the generator of a positive $C_{0}$-semigroup on $L^{p}(\Omega)$ for every open and connected subset $\Omega$ of $\mathbb{R}^{d}$. As we already mentioned in the introduction, the key is a generation result for the case $p=2$ and Kolmogorov kernel estimates. We will prove these two items in this and the following section.

We define the realisation of the Ornstein-Uhlenbeck operator in $L^{2}(\Omega)$ by

$$
\begin{aligned}
D\left(A_{\Omega, 2}\right) & =H_{0}^{1}(\Omega) \cap\left\{u \in H_{\mathrm{loc}}^{2}(\Omega): \mathcal{A} u \in L^{2}(\Omega)\right\}, \\
\left(A_{\Omega, 2} u\right)(x) & =\sum_{i, j=1}^{d} q_{i j} D_{i} D_{j} u(x)+B x \cdot \nabla u(x) \\
& =\sum_{i, j=1}^{d} q_{i j} D_{i} D_{j} u(x)+\sum_{i, j=1}^{d} b_{i j} x_{j} D_{i} u(x), \quad x \in \Omega \subset \mathbb{R}^{d},
\end{aligned}
$$

where $Q=\left(q_{i j}\right)_{i, j=1}^{d} \in \mathbb{R}^{d \times d}$ is a symmetric and positive definite matrix and $B=\left(b_{i j}\right)_{i, j=1}^{d} \in \mathbb{R}^{d \times d} \backslash\{0\}$. Note that this notation is consistent with the definition of $A_{\mathbb{R}^{d}, 2}$ in (2.2). In fact, the domain in (2.2) is clearly contained in the domain given here, so when we have shown in Proposition 3.5 that $A_{\Omega, 2}$ is dissipative, the equality of the two domains follows by the following general observation.

Remark 3.1. Let $B$ be the generator of a $C_{0}$-semigroup on some Banach space $X$ and let $A \supseteq B$ be dissipative. Then we already have $A=B$. In fact, there exists $\lambda>0$, such that $\lambda-B$ is surjective and $\lambda-A$ is injective by dissipativity. Thus the claim follows by [7, IV.1.21 (5)].

Now we can formulate our result for the case $p=2$.

Theorem 3.2. Let $\Omega \subseteq \mathbb{R}^{d}$ be a domain. Then the operator $A_{\Omega, 2}$ generates a positive $C_{0}$-semigroup $\left(T_{\Omega, 2}(t)\right)_{t \geq 0}$ on $L^{2}(\Omega)$ with $\left\|T_{\Omega, 2}(t)\right\|_{\mathcal{L}\left(L^{2}(\Omega)\right)} \leq \mathrm{e}^{-\frac{\operatorname{tr}(B)}{2} t}$ 
for all $t \geq 0$. Moreover for every $\lambda>-\operatorname{tr}(B) / 2$ and every $t \geq 0$ we have the domination properties

$$
\begin{aligned}
\left|R\left(\lambda, A_{\Omega, 2}\right) f\right| & \leq R\left(\lambda, A_{\mathbb{R}^{d}, 2}\right)|\tilde{f}|, & & f \in L^{2}(\Omega), \\
\left|T_{\Omega, 2}(t) f\right| & \leq T_{\mathbb{R}^{d}, 2}(t)|\tilde{f}|=K_{2}(t)|\tilde{f}|, & & f \in L^{2}(\Omega),
\end{aligned}
$$

where $\tilde{f}$ denotes the extension of $f$ by 0 . In particular, $T_{\Omega, 2}$ fulfills a Kolmogorov kernel estimate with $M=1$ and $\omega=0$.

In the following we use the notation

$$
\left(\mathcal{A}_{0} u\right)(x)=\sum_{i, j=1}^{d} q_{i j} D_{i} D_{j} u(x) \quad \text { and } \quad(\mathcal{L} u)(x)=B x \cdot \nabla u(x)
$$

for the diffusion and the drift part of $\mathcal{A}$, respectively. We start with a simple lemma, that will be useful for many proofs.

Lemma 3.3. Let $G \subseteq \mathbb{R}^{d}$ be open and $u \in H_{0}^{1}(G)$ be a real-valued function. For any $\varphi \in C_{c}^{\infty}\left(\mathbb{R}^{d}\right)$ we have

$$
\int_{G}(\mathcal{L} u) u \varphi=-\frac{\operatorname{tr}(B)}{2} \int_{\Omega} u^{2} \varphi-\frac{1}{2} \int_{\Omega} u^{2} \mathcal{L} \varphi
$$

Proof. Since $u \in H_{0}^{1}(G)$, there is a sequence $\left(u_{n}\right)_{n \in \mathbb{N}} \subseteq C_{c}^{\infty}(G)$ converging to $u$ in $H^{1}(G)$. Therefore, we have $\int_{G}(\mathcal{L} u) u \varphi=\lim _{n \rightarrow \infty} \int_{G}\left(\mathcal{L} u_{n}\right) u_{n} \varphi$, since $B x$ is bounded on the support of $\varphi$. We get

$$
\begin{aligned}
\int_{G}\left(\mathcal{L} u_{n}\right) u_{n} \varphi & =\int_{G} B x \cdot \nabla u_{n} u_{n} \varphi=-\int_{G} \operatorname{div}\left(u_{n} \varphi B x\right) u_{n} \\
& =-\int_{G}\left(\mathcal{L} u_{n}\right) u_{n} \varphi-\operatorname{tr}(B) \int_{G} u_{n}^{2} \varphi-\int_{G} u_{n}^{2} \mathcal{L} \varphi
\end{aligned}
$$

Letting $n$ tend to $\infty$, we derive $\int_{G}(\mathcal{L} u) u \varphi=-\frac{\operatorname{tr}(B)}{2} \int_{G} u^{2} \varphi-\frac{1}{2} \int_{G} u^{2} \mathcal{L} \varphi$.

Remark 3.4. To be precise, one has to check that integration by parts is allowed in the proof of Lemma 3.3. This will be used again later on, so it might be useful to note the following generalisation. For any open set $G$, integration by parts is possible if $u \in C_{c}^{\infty}(G)$ and $v \in H_{\mathrm{loc}}^{1}(G)$.

In this case, there is a compact set $K \subseteq G$ with $\operatorname{supp}(u) \subseteq K^{\circ}$. Then $v \in$ $H^{1}(K)$ and by the definition of weak derivatives one gets for all $1 \leq i \leq d$

$$
\int_{G} u\left(D_{i} v\right)=\int_{K} u\left(D_{i} v\right)=-\int_{K}\left(D_{i} u\right) v=-\int_{G}\left(D_{i} u\right) v .
$$


In order to show that $A_{\Omega, 2}$ is a generator of a $C_{0}$-semigroup we will apply the Lumer-Phillips theorem. So we first need dissipativity.

Proposition 3.5. The operator

$$
A:=A_{\Omega, 2}+\frac{\operatorname{tr}(B)}{2}
$$

is dissipative in $L^{2}(\Omega)$.

Proof. Let $f \in D\left(A_{\Omega, 2}\right)=D(A)$. Writing $f=u+i v$ for suitable real $u, v \in$ $L^{2}(\Omega)$, we get

$$
\operatorname{Re} \int_{\Omega}(A f) \bar{f}=\int_{\Omega}(A u) u+\int_{\Omega}(A v) v
$$

so it suffices to show $\int_{\Omega}(A u) u \leq 0$ for real-valued functions $u$. Note that $u, v \in$ $D\left(A_{\Omega, 2}\right)$, since the coefficients of $\mathcal{A}$ are real.

We choose $\eta \in C_{c}^{\infty}\left(\mathbb{R}^{d}\right)$ vanishing outside of $B_{2}(0)$ with $\left.\eta\right|_{B_{1}(0)}=\mathbf{1}$ and define $\eta_{m}(x)=\eta\left(\frac{x}{m}\right)$ for $m \in \mathbb{N}$. Since $(A u) u \in L^{1}(\Omega)$ we derive $\lim _{m \rightarrow \infty} \int_{\Omega}(A u) u \eta_{m}=$ $\int_{\Omega}(A u) u$ by the pointwise convergence of $\eta_{m}$ to 1 and Lebesgue's Theorem.

Next we choose a sequence $\left(u_{n}\right)_{n \in \mathbb{N}}$ of $C_{c}^{\infty}(\Omega)$-functions converging to $u$ with respect to the $H^{1}$-norm. Now, partial integration (as in Remark 3.4) yields

$$
\begin{aligned}
& \int_{\Omega}\left(A_{\Omega, 2} u\right)(x) u_{n}(x) \eta_{m}(x) \mathrm{d} x \\
= & \int_{\Omega} \sum_{i, j=1}^{d} q_{i j} D_{i} D_{j} u(x) u_{n}(x) \eta_{m}(x) \mathrm{d} x+\int_{\Omega} \sum_{i, j=1}^{d} b_{i j} x_{j} D_{i} u(x) u_{n}(x) \eta_{m}(x) \mathrm{d} x \\
= & \int_{\Omega} \sum_{i, j=1}^{d} q_{i j} D_{i} D_{j} u(x) u_{n}(x) \eta_{m}(x) \mathrm{d} x+\frac{1}{2} \int_{\Omega} \sum_{i, j=1}^{d} b_{i j} x_{j} D_{i} u(x) u_{n}(x) \eta_{m}(x) \mathrm{d} x \\
& +\frac{1}{2} \int_{\Omega} \sum_{i, j=1}^{d} b_{i j} x_{j} D_{i} u(x) u_{n}(x) \eta_{m}(x) \mathrm{d} x \\
= & -\int_{\Omega} \sum_{i, j=1}^{d} q_{i j} D_{i} u(x) D_{j} u_{n}(x) \eta_{m}(x) \mathrm{d} x-\int_{\Omega} \sum_{i, j=1}^{d} q_{i j} D_{i} u(x) u_{n}(x) D_{j} \eta_{m}(x) \mathrm{d} x \\
& +\frac{1}{2} \int_{\Omega} \sum_{i, j=1}^{d} b_{i j} x_{j} D_{i} u(x) u_{n}(x) \eta_{m}(x) \mathrm{d} x-\frac{1}{2} \int_{\Omega} \sum_{i, j=1}^{d} b_{i j} x_{j} u(x) D_{i} u_{n}(x) \eta_{m}(x) \mathrm{d} x \\
& -\frac{1}{2} \int_{\Omega} \sum_{i, j=1}^{d} b_{i j} x_{j} u_{n}(x) u(x) D_{i} \eta_{m}(x) \mathrm{d} x-\frac{\operatorname{tr}(B)}{2} \int_{\Omega} u(x) u_{n}(x) \eta_{m}(x) \mathrm{d} x .
\end{aligned}
$$


Note that, thanks to the bounded supports of the functions $\eta_{m}$ and $u_{n}$, all integrals in the above calculations are well defined.

The $H^{1}$-convergence of the sequence $\left(u_{n}\right)_{n \in \mathbb{N}}$ yields

$\lim _{n \rightarrow \infty} \int_{\Omega} \sum_{i, j=1}^{d} q_{i j} D_{i} u(x) D_{j} u_{n}(x) \eta_{m}(x) \mathrm{d} x=\int_{\Omega} \sum_{i, j=1}^{d} q_{i j} D_{i} u(x) D_{j} u(x) \eta_{m}(x) \mathrm{d} x$

and

$$
\lim _{n \rightarrow \infty} \int_{\Omega} \sum_{i, j=1}^{d} b_{i j} x_{j} D_{i} u_{n}(x) u(x) \eta_{m}(x) \mathrm{d} x=\int_{\Omega} \sum_{i, j=1}^{d} b_{i j} x_{j} D_{i} u(x) u(x) \eta_{m}(x) \mathrm{d} x,
$$

since $x_{j} \eta_{m}(x)$ is bounded. The other summands can be treated analogously, so we derive

$$
\begin{aligned}
& \int_{\Omega}\left(A_{\Omega, 2} u\right)(x) u(x) \eta_{m}(x) \mathrm{d} x=\lim _{n \rightarrow \infty} \int_{\Omega}\left(A_{\Omega, 2} u\right)(x) u_{n}(x) \eta_{m}(x) \mathrm{d} x \\
= & -\int_{\Omega} \sum_{i, j=1}^{d} q_{i j} D_{i} u(x) D_{j} u(x) \eta_{m}(x) \mathrm{d} x-\int_{\Omega} \sum_{i, j=1}^{d} q_{i j} D_{i} u(x) u(x) D_{j} \eta_{m}(x) \mathrm{d} x \\
& -\frac{1}{2} \int_{\Omega} \sum_{i, j=1}^{d} b_{i j} x_{j} u(x) u(x) D_{i} \eta_{m}(x) \mathrm{d} x-\frac{\operatorname{tr}(B)}{2} \int_{\Omega} u(x) u(x) \eta_{m}(x) \mathrm{d} x .
\end{aligned}
$$

Next, we want to pass to the limit $m \rightarrow \infty$, so we have to consider the terms containing derivatives of $\eta_{m}$. The equality $\left(D_{i} \eta_{m}\right)(x)=\frac{1}{m}\left(D_{i} \eta\right)\left(\frac{x}{m}\right)$ implies

$$
x_{j} D_{i} \eta_{m}(x)=\frac{x_{j}}{m} D_{i} \eta\left(\frac{x}{m}\right)=0 \text { for } \frac{|x|}{m}>2 \text {, }
$$

and therefore all functions $x_{j} D_{i} \eta_{m}(x), 1 \leq i, j \leq d$, are bounded with

$$
\left|x_{j} D_{i} \eta_{m}(x)\right|=\left|\frac{x_{j}}{m} D_{i} \eta\left(\frac{x}{m}\right)\right|<2\|\nabla \eta\|_{\infty}
$$

and have support inside $\left\{x \in \mathbb{R}^{d}: m \leq|x| \leq 2 m\right\}$.

Now let $\varepsilon>0$. Since $u^{2} \in L^{1}(\Omega)$, there is a compact subset $K_{\varepsilon} \subseteq \Omega$ with

$$
\int_{\Omega \backslash K_{\varepsilon}} u^{2} \leq \frac{\varepsilon}{2\|\nabla \eta\|_{\infty}}
$$

If we choose $m_{0}$ large enough, we have $K_{\varepsilon} \cap \operatorname{supp}\left(x_{j} D_{i} \eta_{m}\right)=\emptyset$ for all $m \geq m_{0}$ and therefore

$$
\left|\int_{\Omega} u^{2}(x) x_{j} D_{i} \eta_{m}(x) \mathrm{d} x\right|=\left|\int_{\Omega \backslash K_{\varepsilon}} u^{2}(x) x_{j} D_{i} \eta_{m}(x) \mathrm{d} x\right| \leq \varepsilon .
$$


This proves

$$
\lim _{m \rightarrow \infty} \int_{\Omega} \sum_{i, j=1}^{d} b_{i j} x_{j} u(x) u(x) D_{i} \eta_{m}(x) \mathrm{d} x=0 .
$$

Since $\left\|D_{i} \eta_{m}\right\|_{\infty} \leq \frac{1}{m}\|\nabla \eta\|_{\infty}$, we also have

$$
\lim _{m \rightarrow \infty} \int_{\Omega} \sum_{i, j=1}^{d} q_{i j} D_{i} u(x) u(x) D_{j} \eta_{m}(x) \mathrm{d} x=0 .
$$

Finally we obtain dissipativity of $A$ by

$$
\begin{aligned}
\int_{\Omega}(A u) u & =\lim _{m \rightarrow \infty} \int_{\Omega}\left(A_{\Omega, 2} u+\frac{\operatorname{tr}(B)}{2} u\right) u \eta_{m} \\
& =-\int_{\Omega} \sum_{i, j=1}^{d} q_{i j} D_{i} u(x) D_{j} u(x) \mathrm{d} x \leq 0,
\end{aligned}
$$

as $Q$ is positive definite.

In order to show that $A_{\Omega, 2}$ is a generator, it remains to be proven that $\lambda-A$ is surjective for some fixed $\lambda>0$. This will be done by approximating the solution $u$ of the resolvent problem $(\lambda-A) u=f, f \in L^{2}(\Omega)$, with solutions of the same problem on bounded and regular subdomains of $\Omega$.

By [5, II.4, Lemma 1], there exists an increasing sequence $\left(\Omega_{n}\right)_{n \in \mathbb{N}}$ of bounded subdomains of $\Omega$, that have a $C^{2}$-boundary, such that $\Omega=\bigcup_{n \in \mathbb{N}} \Omega_{n}$. Note that the specific choice of this sequence is not important, since by dissipativity of $A$ a solution of the resolvent problem is unique, whenever it exists.

Since the coefficients of $\mathcal{L}$ are bounded on bounded sets, by standard perturbation theory, the operator $\mathcal{A}+\operatorname{tr}(B) / 2$ generates a $C_{0}$-semigroup on $L^{2}\left(\Omega_{n}\right)$ for every $n \in \mathbb{N}$, when we equip it with the domain $D=H_{0}^{1}\left(\Omega_{n}\right) \cap H^{2}\left(\Omega_{n}\right)$. As $D\left(A_{\Omega_{n}, 2}\right)$ contains $D$, we get $D\left(A_{\Omega_{n}, 2}\right)=D$ again by Remark 3.1. This coincidence of the domains even gives us some more precious information. Since the generator $A_{\Omega_{n}, 2}+\operatorname{tr}(B) / 2$ is dissipative it even generates a contraction semigroup on $L^{2}\left(\Omega_{n}\right)$ for every $n \in \mathbb{N}$. Thus the bounds on the resolvent do not depend on $n$, which will be important in the following.

Fixing $\lambda>0$ and $f \in L^{2}(\Omega)$ we find a unique solution $u_{n} \in H_{0}^{1}\left(\Omega_{n}\right) \cap$ $H^{2}\left(\Omega_{n}\right)$ for the problem $\lambda u_{n}-A_{\Omega_{n}, 2} u_{n}-\frac{\operatorname{tr}(B)}{2} u_{n}=\left.f\right|_{\Omega_{n}}$ for every $n \in \mathbb{N}$ with

$$
\left\|u_{n}\right\|_{L^{2}\left(\Omega_{n}\right)}=\left\|\left.R\left(\lambda, A_{\Omega_{n}, 2}+\frac{\operatorname{tr}(B)}{2}\right) f\right|_{\Omega_{n}}\right\|_{L^{2}\left(\Omega_{n}\right)} \leq \frac{1}{\lambda}\|f\|_{L^{2}(\Omega)},
$$

independently of the domain $\Omega_{n}$. 
As by [1, Lemma 3.22], the trivial extension of $u_{n}$ is an element of $H^{1}\left(\mathbb{R}^{d}\right)$ and hence of $H_{0}^{1}(\Omega)$, we may regard $\left(u_{n}\right)_{n \in \mathbb{N}}$ as a sequence in $H_{0}^{1}(\Omega)$. The next lemma will show that even the gradients of the functions $u_{n}$ are bounded independently of the chosen domain, which does not follow by elliptic regularity alone.

Lemma 3.6. Let $G \subseteq \mathbb{R}^{d}$ be a bounded domain with $C^{2}$-boundary and $u \in H_{0}^{1}(G) \cap$ $H^{2}(G)$ be a solution of $\lambda u-A_{G, 2} u-\frac{\operatorname{tr}(B)}{2} u=g$ for some $\lambda>0$. Then

$$
\|\nabla u\|_{2} \leq \sqrt{\frac{2}{\lambda}}\left\|Q^{-\frac{1}{2}}\right\|\|g\|_{2} .
$$

Proof. Let $u=v+i w$ with real-valued $v, w \in H_{0}^{1}(G) \cap H^{2}(G)$. Then

$$
\begin{aligned}
\left\|D^{\alpha} u\right\|_{2}^{2}=\int_{G} D^{\alpha} u \overline{D^{\alpha} u} & =\int_{G}\left(D^{\alpha} v+i D^{\alpha} w\right)\left(D^{\alpha} v-i D^{\alpha} w\right) \\
& =\left\|D^{\alpha} v\right\|_{2}^{2}+\left\|D^{\alpha} w\right\|_{2}^{2}
\end{aligned}
$$

for any multiindex $\alpha$ with $|\alpha| \leq 2$.

$$
\begin{aligned}
& \int_{G} Q^{\frac{1}{2}} \nabla v Q^{\frac{1}{2}} \nabla v=\int_{G} Q \nabla v \nabla v=-\int_{G}\left(\mathcal{A}_{0} v\right) v \\
= & \int_{G}\left(\lambda v-A_{G, 2} v-\frac{\operatorname{tr}(B)}{2} v\right) v-\lambda \int_{G} v v+\int_{G}(\mathcal{L} v) v+\frac{\operatorname{tr}(B)}{2} \int_{G} v v \\
= & \int_{G}\left(\lambda v-A_{G, 2} v-\frac{\operatorname{tr}(B)}{2} v\right) v-\lambda \int_{G} v v \\
= & \int_{G} \operatorname{Re}(g) R\left(\lambda, A_{G, 2}+\frac{\operatorname{tr}(B)}{2}\right) \operatorname{Re}(g)-\lambda\left\|R\left(\lambda, A_{G, 2}+\frac{\operatorname{tr}(B)}{2}\right) \operatorname{Re}(g)\right\|_{2}^{2} .
\end{aligned}
$$

We conclude by (3.1)

$$
\|\nabla v\|_{2}^{2}=\left\|Q^{-\frac{1}{2}}\left(Q^{\frac{1}{2}} \nabla v\right)\right\|_{2}^{2} \leq\left\|Q^{-\frac{1}{2}}\right\|^{2} \frac{2}{\lambda}\|\operatorname{Re}(g)\|_{2}^{2} .
$$

Repeating the same calculations for $w$, we obtain

$$
\|\nabla u\|_{2}^{2} \leq\left\|Q^{-\frac{1}{2}}\right\|^{2} \frac{2}{\lambda}\|\operatorname{Re}(g)\|_{2}^{2}+\left\|Q^{-\frac{1}{2}}\right\|^{2} \frac{2}{\lambda}\|\operatorname{Im}(g)\|_{2}^{2}=\left\|Q^{-\frac{1}{2}}\right\|^{2} \frac{2}{\lambda}\|g\|_{2}^{2} .
$$

Now we can prove the main result of this section.

Proposition 3.7. Let $\Omega \subseteq \mathbb{R}^{d}$ be a domain. Then $A_{\Omega, 2}$ is the generator of a $C_{0}$ semigroup $\left(T_{\Omega, 2}(t)\right)_{t \geq 0}$ on $L^{2}(\Omega)$ with $\left\|T_{\Omega, 2}(t)\right\| \leq \mathrm{e}^{-\frac{\operatorname{tr}(B)}{2} t}$. 
Proof. It only remains to show that for a fixed $\lambda>0$ and for every $f \in L^{2}(\Omega)$ there exists a function $u \in H_{0}^{1}(\Omega) \cap\left\{v \in H_{\mathrm{loc}}^{2}(\Omega): \mathcal{A} v \in L^{2}(\Omega)\right\}$ with $\lambda u-A u=f$.

We consider the sequence $\left(u_{n}\right)_{n \in \mathbb{N}}$ of trivial extensions of the solutions on $\Omega_{n}$ mentioned above. In view of Lemma 3.6, it is bounded in $H_{0}^{1}(\Omega)$, so there exists a weakly convergent subsequence $\left(u_{n_{k}}\right)_{k \in \mathbb{N}}$. We denote its limit by $u$ and show in the following that $u$ is the desired solution.

As a first step, we will prove that $u \in H_{\text {loc }}^{2}(\Omega)$. Fix two compact sets $K_{1}, K_{2} \subseteq$ $\Omega$ with $K_{1} \subseteq K_{2}^{\circ}$. Then, by construction, $K_{2} \subseteq \Omega_{n_{k}}$ for sufficiently large $k$. The coefficients of $\mathcal{A}$ are bounded on $K_{2}$, so [11, Theorem 9.11] implies that there is a constant $C$ depending on $K_{1}, K_{2}$ and the bound of the coefficients on $K_{2}$, such that $\left\|u_{n_{k}}\right\|_{H^{2}\left(K_{1}\right)} \leq C\left(\left\|u_{n_{k}}\right\|_{2}+\|f\|_{2}\right)$, so there exists a weakly convergent subsequence $\left(u_{n_{k_{l}}}\right)_{l \in \mathbb{N}}$ in $H^{2}\left(K_{1}\right)$. Let $v$ denote its limit. Since the sequence $\left(\left.u_{n_{k_{l}}}\right|_{K_{1}}\right)_{l \in \mathbb{N}}$ also converges weakly to $\left.u\right|_{K_{1}}$ in $L^{2}\left(K_{1}\right)$, we derive $v=\left.u\right|_{K_{1}}$ from the uniqueness of weak limits in $L^{2}\left(K_{1}\right)$. This shows $u \in H_{\text {loc }}^{2}(\Omega)$.

In order to finish the proof, it remains to show $\lambda u-A u=f$. Let $g \in C_{c}^{\infty}(\Omega)$ and fix a compactum $K \subseteq \Omega$ with $\operatorname{supp}(g) \subseteq K^{\circ}$. Then $K \subseteq \Omega_{n_{k}}$ for $k$ large enough. Let $\left(u_{n_{k_{l}}}\right)_{l \in \mathbb{N}}$ be again a weakly convergent subsequence of $\left(u_{n_{k}}\right)_{k \in \mathbb{N}}$ on $H^{2}(K)$ and $A^{\star}$ be the formal adjoint of $A$. Then we conclude

$$
\begin{aligned}
& \int_{\Omega}(\lambda u-A u-f) g=\int_{K}(\lambda u-A u-f) g=\int_{K}\left(\lambda u g-u A^{\star} g-f g\right) \\
= & \lim _{l \rightarrow \infty} \int_{K}\left(\lambda u_{n_{k_{l}}} g-u_{n_{k_{l}}} A^{\star} g-f g\right)=\lim _{l \rightarrow \infty} \int_{K}\left(\lambda u_{n_{k_{l}}}-A u_{n_{k_{l}}}-f\right) g=0 .
\end{aligned}
$$

Thus the assertion follows by the fundamental theorem of variational calculus.

\section{Domination and positivity of the semigroup}

The aim of this section is to prove Kolmogorov kernel estimates for the semigroup $\left(T_{\Omega, 2}(t)\right)_{t \geq 0}$ obtained in Proposition 3.7. While doing so, we also obtain positivity of the semigroup. Our method is inspired by the proof of heat kernel estimates for the Dirichlet Laplacian, cf. [2].

Lemma 4.1. Let $\Omega \subseteq \mathbb{R}^{d}$ be a domain, $\lambda>-\operatorname{tr}(B) / 2$ and let $u \in D\left(A_{\Omega, 2}\right)$, $v \in H^{1}(\Omega) \cap\left\{f \in \bar{H}_{\mathrm{loc}}^{2}(\Omega): \mathcal{A} f \in L^{2}(\Omega)\right\}$ be real-valued functions such that $v \geq 0$. Then the inequality $(\lambda-\mathcal{A}) u \leq(\lambda-\mathcal{A}) v$ a.e. implies $u \leq v$ a.e.

Proof. As in the proof of Proposition 3.5, we choose a positive $\eta \in C_{c}^{\infty}\left(\mathbb{R}^{d}\right)$ vanishing outside of $B_{2}(0)$ with $\left.\eta\right|_{B_{1}(0)}=\mathbf{1}$ and define $\eta_{m}(x)=\eta\left(\frac{x}{m}\right)$ for $m \in \mathbb{N}$.

By hypotheses, we have

$$
\lambda(u-v)-\mathcal{A}_{0}(u-v)-\mathcal{L}(u-v) \leq 0, \quad \text { a.e. }
$$


so we obtain

$$
\lambda \int_{\Omega}(u-v) \varphi \eta_{m}-\sum_{i, j=1}^{d} \int_{\Omega} q_{i j} D_{i} D_{j}(u-v) \varphi \eta_{m}-\int_{\Omega} B x \cdot \nabla(u-v) \varphi \eta_{m} \leq 0
$$

for all $m \in \mathbb{N}$ and all positive $\varphi \in C_{c}^{\infty}(\Omega)$. By integration by parts, cf. Remark 3.4, we conclude that

$$
\begin{aligned}
\lambda \int_{\Omega}(u-v) \varphi \eta_{m} & +\sum_{i, j=1}^{d} \int_{\Omega} q_{i j} D_{j}(u-v) D_{i} \varphi \eta_{m}+\sum_{i, j=1}^{d} \int_{\Omega} q_{i j} D_{j}(u-v) \varphi D_{i} \eta_{m} \\
& -\int_{\Omega} B x \cdot \nabla(u-v) \eta_{m} \varphi \leq 0 .
\end{aligned}
$$

for all $m \in \mathbb{N}$. Now, this last inequality is even valid for all $\varphi \in H_{0}^{1}(\Omega)_{+}$by density.

In the following we show that $(u-v)^{+} \in H_{0}^{1}(\Omega)_{+}$. In order to do so, we choose a sequence $\left(u_{n}\right) \subseteq C_{c}^{\infty}(\Omega)$, that converges to $u$ in $H^{1}(\Omega)$. Then the function $\left(u_{n}-v\right)^{+}$is in $H^{1}(\Omega)_{+}$for every $n \in \mathbb{N}$ and since $v \geq 0$, we get $\operatorname{supp}\left(\left(u_{n}-v\right)^{+}\right) \subseteq$ $\operatorname{supp}\left(u_{n}\right)$. Thus $\left(u_{n}-v\right)^{+}$has compact support in $\Omega$, which implies $\left(u_{n}-v\right)^{+} \in$ $H_{0}^{1}(\Omega)_{+}$. This finally yields $(u-v)^{+} \in H_{0}^{1}(\Omega)_{+}$, as $H_{0}^{1}(\Omega)$ is a closed subspace of $H^{1}(\Omega)$.

Putting $\varphi=(u-v)^{+}$in the above inequality and observing that then all the integrals vanish on the set $\{u \leq v\}$, we get

$$
\begin{aligned}
& \lambda \int_{\Omega}\left((u-v)^{+}\right)^{2} \eta_{m}+\int_{\Omega} \nabla(u-v)^{+} Q \nabla(u-v)^{+} \eta_{m} \\
& -\int_{\Omega} B x \cdot \nabla(u-v)^{+}(u-v)^{+} \eta_{m}+\sum_{i, j=1}^{n} \int_{\Omega} q_{i j} D_{j}(u-v)^{+}(u-v)^{+} D_{i} \eta_{m} \leq 0 .
\end{aligned}
$$

Now Lemma 3.3 yields for the third integral

$$
\begin{aligned}
\int_{\Omega} B x \cdot \nabla(u-v)^{+}(u-v)^{+} \eta_{m}= & -\frac{\operatorname{tr}(B)}{2} \int_{\Omega}\left((u-v)^{+}\right)^{2} \eta_{m} \\
& -\int_{\Omega} B x \cdot \nabla \eta_{m}\left((u-v)^{+}\right)^{2} .
\end{aligned}
$$

As all the limits for $m \rightarrow \infty$ on the left hand side exist (cf. proof of Proposition 3.5), this implies

$$
-\int_{\Omega} B x \cdot \nabla(u-v)^{+}(u-v)^{+}=\frac{\operatorname{tr}(B)}{2} \int_{\Omega}\left((u-v)^{+}\right)^{2} .
$$

We derive the inequality

$$
\left(\lambda+\frac{\operatorname{tr}(B)}{2}\right) \int_{\Omega}\left((u-v)^{+}\right)^{2}+\int_{\Omega} \nabla(u-v)^{+} Q \nabla(u-v)^{+} \leq 0 .
$$


Since $Q$ is positive definite and $\lambda>-\operatorname{tr}(B) / 2$, this can only be true, if both integrals are zero, in particular, we get $\left\|(u-v)^{+}\right\|_{L^{2}(\Omega)}=0$, which implies $u \leq v$ a.e. for $\mathcal{A}$.

Setting $v=0$, as an immediate corollary, we get the following positivity result

Corollary 4.2. Let $\lambda>-\operatorname{tr}(B) / 2$ and let $u \in D\left(A_{\Omega, 2}\right)$ be a real-valued function. Then

$$
\begin{aligned}
& (\lambda-\mathcal{A}) u \leq 0 \text { a.e. } \Longrightarrow u \leq 0 \text { a.e. and } \\
& (\lambda-\mathcal{A}) u \geq 0 \text { a.e. } \Longrightarrow u \geq 0 \text { a.e. }
\end{aligned}
$$

The next step is to show that the resolvent and the semigroup act monotonously, when the domain $\Omega$ is enlarged. As a byproduct of the proof, we also deduce the positivity of the semigroup and the resolvent.

Proposition 4.3. Let $\Omega_{1}, \Omega_{2} \subseteq \mathbb{R}^{d}$ be domains and let $\Omega_{1} \subseteq \Omega_{2}$. Then for every $f \in L^{2}\left(\Omega_{1}\right)_{+}$, for every $\lambda>-\operatorname{tr}(B) / 2$ and every $t \geq 0$, we have

1. $0 \leq R\left(\lambda, A_{\Omega_{1}, 2}\right) f \leq R\left(\lambda, A_{\Omega_{2}, 2}\right) \tilde{f}$,

2. $0 \leq T_{\Omega_{1}, 2}(t) f \leq T_{\Omega_{2}, 2}(t) \tilde{f}$

almost everywhere. Here $\tilde{f}$ denotes the extension of $f$ by 0 .

Proof.

1. Put $u:=R\left(\lambda, A_{\Omega_{1}, 2}\right) f$ and $v:=R\left(\lambda, A_{\Omega_{2}, 2}\right) \tilde{f}$. Then, both $u$ and $v$ are realvalued. Indeed, for $u=u_{1}+i u_{2}$, we have $f=\left(\lambda-A_{\Omega_{1}, 2}\right) u=\left(\lambda-A_{\Omega_{1}, 2}\right) u_{1}+$ $i\left(\lambda-A_{\Omega_{1}, 2}\right) u_{2}$, where $\left(\lambda-A_{\Omega_{1}, 2}\right) u_{j}, j=1,2$, are real-valued functions. This implies $\left(\lambda-A_{\Omega_{1}, 2}\right) u_{2}=0$, and, by injectivity of $\left(\lambda-A_{\Omega_{1}, 2}\right)$, even $u_{2}=0$. The argument for $v$ is the same.

Since $u \in D\left(A_{\Omega_{1}, 2}\right), v \in D\left(A_{\Omega_{2}, 2}\right)$ and $f$ and $\tilde{f}$ are positive we get $u, v \geq 0$ a.e. by Corollary 4.2. It remains to show $u \leq v$. As $(\lambda-\mathcal{A}) u=f=(\lambda-\mathcal{A}) v$ a.e. in $\Omega_{1}$, we have in particular $(\lambda-\mathcal{A}) u \leq(\lambda-\mathcal{A}) v$. Furthermore, we know that $u \in D\left(A_{\Omega_{1}, 2}\right)$ and the restriction of $v$ to $\Omega_{1}$ is in $H^{1}\left(\Omega_{1}\right) \cap\left\{f \in H_{\mathrm{loc}}^{2}\left(\Omega_{1}\right)\right.$ : $\left.\mathcal{A} f \in L^{2}\left(\Omega_{1}\right)\right\}$. Thus we may apply Lemma 4.1 , in order to get $u \leq v$ a.e. in $\Omega_{1}$.

2. Since $R\left(\lambda, A_{\Omega_{j}, 2}\right)=\frac{1}{\lambda}\left(I-\frac{1}{\lambda} A_{\Omega_{j}, 2}\right)^{-1}, j=1,2$, the first part of the proof yields

$$
0 \leq\left(I-s A_{\Omega_{1}, 2}\right)^{-1} f \leq\left(I-s A_{\Omega_{2}, 2}\right)^{-1} \tilde{f}
$$

for all $0<s<2 /|\operatorname{tr}(B)|$ or for every $s>0$ if $\operatorname{tr}(B)=0$. Thus we have the same for every power $k \in \mathbb{N}$ :

$$
0 \leq\left(I-s A_{\Omega_{1}, 2}\right)^{-k} f \leq\left(I-s A_{\Omega_{2}, 2}\right)^{-k} \tilde{f} .
$$


Now let $t>0$. Then, if $k$ is large enough, we have $t / k<2 /|\operatorname{tr}(B)|$ and

$$
0 \leq\left(I-\frac{t}{k} A_{\Omega_{1}, 2}\right)^{-k} f \leq\left(I-\frac{t}{k} A_{\Omega_{2}, 2}\right)^{-k} \tilde{f} .
$$

Passing to the limit $k \rightarrow \infty$, the claim follows (cf. [7, Cor. III.5.5]).

Since for a positive operator $T$ on a Banach lattice $X$ we always have the inequality $|T f| \leq T|f|$ for all $f \in X$ (cf. [19, II, §2 and §11]), for arbitrary $f \in L^{2}\left(\Omega_{1}\right)$ we get:

Corollary 4.4. If $\Omega_{1}$ and $\Omega_{2}$ are as in Proposition 4.3, we have for every $f \in$ $L^{2}\left(\Omega_{1}\right)$, for every $\lambda>-\operatorname{tr}(B) / 2$ and every $t \geq 0$

1. $\left|T_{\Omega_{1}, 2}(t) f\right| \leq T_{\Omega_{2}, 2}(t)|\tilde{f}|$,

2. $\left|R\left(\lambda, A_{\Omega_{1}, 2}\right) f\right| \leq R\left(\lambda, A_{\left.\Omega_{2}, 2\right)}\right)|\tilde{f}|$.

Setting $\Omega_{2}=\mathbb{R}^{d}$ in Corollary 4.4, we have finally completed the proof of Theorem 3.2.

Now we have shown that $A_{\Omega, 2}$ satisfies a Kolmogorov kernel estimate with $M=1$ and $\omega=0$, so the results stated in Chapter 2 hold true. We collect them in the following theorem.

Theorem 4.5. Let $\Omega \subseteq \mathbb{R}^{d}$ be a domain. Then $\left(T_{\Omega, p}(t)\right)_{t \geq 0}, 1 \leq p<\infty$, is a family of consistent, positive $C_{0}$-semigroups on $L^{p}(\Omega)$ with $\left\|T_{\Omega, p}(t)\right\|_{\mathcal{L}\left(L^{p}(\Omega)\right)} \leq$ $\mathrm{e}^{-\frac{\mathrm{tr}(B)}{p} t}$ for all $1 \leq p<\infty$ and all $t \geq 0$. We denote by $A_{\Omega, p}$ the generator of $T_{\Omega, p}(t)$. Then, for every $\lambda>-\operatorname{tr}(B) / p$ and every $t>0$ we have the domination properties

$$
\begin{aligned}
\left|R\left(\lambda, A_{\Omega, p}\right) f\right| & \leq R\left(\lambda, A_{\mathbb{R}^{d}, p}\right)|\tilde{f}|, & & f \in L^{p}(\Omega), \\
\left|T_{\Omega, p}(t) f\right| & \leq T_{\mathbb{R}^{d}, p}(t)|\tilde{f}|=\left(k_{t} *|\tilde{f}|\right)\left(e^{t B} \cdot\right), & & f \in L^{p}(\Omega),
\end{aligned}
$$

where $\tilde{f}$ denotes the extension of $f$ by 0 . Moreover, the operators $A_{\Omega, p}-|\operatorname{tr}(B)|$ admit a bounded $H^{\infty}$-calculus for every $1<p<\infty$ and we have

1. $R\left(\lambda, A_{\Omega, p}\right) f=R\left(\lambda, A_{\Omega, q}\right) f$ for all $f \in L^{p}(\Omega) \cap L^{q}(\Omega)$, all $1 \leq p, q<\infty$ and all $\lambda \in \mathbb{C}$ with $\operatorname{Re}(\lambda)>\max \left(-\frac{\operatorname{tr}(B)}{p},-\frac{\operatorname{tr}(B)}{q}\right)$.

2. The set $\left\{f \in D\left(A_{\Omega, 2}\right) \cap L^{p}(\Omega): A_{\Omega, 2} f \in L^{p}(\Omega)\right\}$ is contained in $D\left(A_{\Omega, p}\right)$ and $A_{\Omega, p} f=A_{\Omega, 2} f$ for all such $f$ and $1 \leq p<\infty$.

\section{The spectrum of $A_{\Omega, p}$ on exterior domains}

We finally turn our attention to the special case of an exterior domain, i.e. $\Omega=$ $\mathbb{R}^{d} \backslash K$ for some compact set $K \subseteq \mathbb{R}^{d}$ with $C^{1,1}$-boundary. Scaling the set $K$ 
down to $\{0\}$, we show that the spectral behaviour of $A_{\Omega, p}$ is the same as in the case $\Omega=\mathbb{R}^{d}$. This means that the spectrum of the drift operator $\mathcal{L}$ on $\mathbb{R}^{d}$, i.e. the whole vertical line $-\operatorname{tr}(B) / p+i \mathbb{R}$ (or in the case $\operatorname{tr}(B)=0$ at least an unbounded subgroup of it) is contained in $\sigma\left(A_{\Omega, p}\right)$. Thus the semigroup $\left(T_{\Omega, p}(t)\right)_{t \geq 0}$ is not eventually norm-continuous. Nevertheless, we have that the spectral bound of $A_{\Omega, p}$ and the growth bound of $T_{\Omega, p}$ coincide and $s\left(A_{\Omega, p}\right)=\omega_{0}\left(T_{\Omega, p}\right)=-\operatorname{tr}(B) / p$. Before we can formulate this theorem, we have to introduce the realisation $L_{\mathbb{R}^{d}, p}$ of $\mathcal{L}$ in $L^{p}\left(\mathbb{R}^{d}\right)$ :

$$
D\left(L_{\mathbb{R}^{d}, p}\right)=\left\{u \in L^{p}\left(\mathbb{R}^{d}\right): \mathcal{L} u \in L^{p}\left(\mathbb{R}^{d}\right)\right\}, \quad L_{\mathbb{R}^{d}, p} u=\mathcal{L} u,
$$

where $\mathcal{L} u$ is understood in the sense of distributions.

We collect the information on the operators $L_{\mathbb{R}^{d}, p}$ that we need in the next proposition. For proofs, see [15].

Proposition 5.1. Let $1 \leq p<\infty$. Then the following holds:

1. The operator $L_{\mathbb{R}^{d}, p}$ is the generator of a $C_{0}$-semigroup $(S(t))_{t \geq 0}$ on $L^{p}\left(\mathbb{R}^{d}\right)$, given by $(S(t) f)(x)=f\left(\mathrm{e}^{t B} x\right), x \in \mathbb{R}^{d}$, for every $f \in L^{p}\left(\mathbb{R}^{d}\right)$.

2. If $\operatorname{tr}(B) \neq 0$, then $\sigma\left(L_{\mathbb{R}^{d}, p}\right)=-\operatorname{tr}(B) / p+i \mathbb{R}$.

3. If $\operatorname{tr}(B)=0$, then $\sigma\left(L_{\mathbb{R}^{d}, p}\right)$ is an additive subgroup of $i \mathbb{R}$, that is not $\{0\}$.

Now the result that we want to prove in this section can be formulated as follows.

Theorem 5.2. Let $K \subset \mathbb{R}^{d}$ be compact with a $C^{1,1}$-boundary and $\Omega=\mathbb{R}^{d} \backslash K$. Then for every $1<p<\infty$ we have

$$
D\left(A_{\Omega, p}\right)=W_{0}^{1, p}(\Omega) \cap W^{2, p}(\Omega) \cap\left\{f \in L^{p}(\Omega): \mathcal{L} f \in L^{p}(\Omega)\right\} .
$$

Furthermore we have the inclusion $\sigma\left(L_{\mathbb{R}^{d}, p}\right) \subseteq \sigma\left(A_{\Omega, p}\right)$ and $s\left(A_{\Omega, p}\right)=\omega_{0}\left(T_{\Omega, p}\right)=$ $-\operatorname{tr}(B) / p$.

Note, that the whole picture changes completely for bounded domains as the semigroup then becomes analytic. It remains however an open question whether Theorem 5.2 is still valid for more general unbounded domains.

Remark 5.3. If $\Omega$ is an exterior domain with $C^{1,1}$-boundary, it was shown in [10] that the Ornstein-Uhlenbeck operator equipped with the domain $W_{0}^{1, p}(\Omega) \cap$ $W^{2, p}(\Omega) \cap\left\{f \in L^{p}(\Omega): \mathcal{L} f \in L^{p}(\Omega)\right\}$ generates a $C_{0}$-semigroup for every $1<p<\infty$ (in fact, this result is formulated for the case $Q=I$, but the proof directly carries over to our situation). For the following results, it is necessary to check that $A_{\Omega, p}$ coincides with this operator, which we will denote by $\tilde{A}_{p}$. For $p=2$, the inclusion $D\left(\tilde{A}_{2}\right) \subseteq D\left(A_{\Omega, 2}\right)$ is clear, so we get $D\left(\tilde{A}_{2}\right)=D\left(A_{\Omega, 2}\right)$ by Remark 3.1.

The construction of the semigroups in [10] now immediately yields consistency. From this we deduce that the semigroups generated by $\tilde{A}_{p}$ and $A_{\Omega, p}$ and hence their generators coincide for $1<p<\infty$. 
In the following we often identify functions $f \in C_{c}^{\infty}\left(\mathbb{R}^{d} \backslash\{0\}\right)$ with their extension by $f(0)=0$ and thus view $C_{c}^{\infty}\left(\mathbb{R}^{d} \backslash\{0\}\right)$ as a subspace of $C_{c}^{\infty}\left(\mathbb{R}^{d}\right)$. The importance of the space $C_{c}^{\infty}\left(\mathbb{R}^{d} \backslash\{0\}\right)$ for the following is due to the following lemma.

Lemma 5.4. The subspace $C_{c}^{\infty}\left(\mathbb{R}^{d} \backslash\{0\}\right) \subseteq D\left(L_{\mathbb{R}^{d}, p}\right)$ is a core for $L_{\mathbb{R}^{d}, p}$.

Proof. Let $f \in C_{c}^{\infty}\left(\mathbb{R}^{d} \backslash\{0\}\right)$ and $t>0$. Then $0 \notin \operatorname{supp}(f)$. In view of the linearity, bijectivity and continuity of the map $x \mapsto \mathrm{e}^{-t B} x$, we conclude that $\operatorname{supp}(S(t) f)=$ $\mathrm{e}^{-t B} \operatorname{supp}(f)$ is a compact set not containing 0 , hence $S(t) f \in C_{c}^{\infty}\left(\mathbb{R}^{d} \backslash\{0\}\right)$ for every $t \geq 0$. Moreover, $C_{c}^{\infty}\left(\mathbb{R}^{d} \backslash\{0\}\right)$ is dense in $L^{p}\left(\mathbb{R}^{d}\right)$, so the lemma follows from [7, Proposition I.1.7].

Now, if $\Omega=\mathbb{R}^{d} \backslash K$ for some compact set $K \subseteq \mathbb{R}^{d}$ with $C^{1,1}$-boundary, for every $k \in \mathbb{N}$ we set

$$
\Omega_{k}:=\left\{x \in \mathbb{R}^{d}: k x \in \Omega\right\}=\mathbb{R}^{d} \backslash \frac{1}{k} K
$$

and consider the operator

$$
A_{k}=\frac{1}{k^{2}} \sum_{i, j=1}^{d} q_{i j} D_{i} D_{j}+\mathcal{L}
$$

with domain

$$
D\left(A_{k}\right)=W_{0}^{1, p}\left(\Omega_{k}\right) \cap W^{2, p}\left(\Omega_{k}\right) \cap\left\{f \in L^{p}\left(\Omega_{k}\right): \mathcal{L} f \in L^{p}\left(\Omega_{k}\right)\right\}
$$

for some given $p \in(1, \infty)$.

By Remark 5.3 we have $D\left(A_{k}\right)=D\left(A_{\Omega_{k}, p}\right)$, so Theorem 4.5 implies that $\left\{\lambda \in \mathbb{C}: \operatorname{Re}(\lambda)>-\frac{\operatorname{tr}(B)}{p}\right\} \subseteq \varrho\left(A_{k}\right)$ for all $k \in \mathbb{N}$. Thus we may compare the resolvents of $A_{k}$ with the resolvent of $A_{\Omega, p}=A_{1}$ for all these $\lambda$. We adapt a technique introduced in [6] to obtain the following result.

Lemma 5.5. We have $\left\|R\left(\lambda, A_{k}\right)\right\|_{\mathcal{L}\left(L^{p}\left(\Omega_{k}\right)\right)}=\left\|R\left(\lambda, A_{\Omega, p}\right)\right\|_{\mathcal{L}\left(L^{p}(\Omega)\right)}$ for every $k \in$ $\mathbb{N}$ and every $\lambda \in \mathbb{C}$ with $\operatorname{Re}(\lambda)>-\frac{\operatorname{tr}(B)}{p}$.

Proof. We consider the map

$$
V_{k}: L^{p}\left(\Omega_{k}\right) \rightarrow L^{p}(\Omega), \quad V_{k}(f)(x)=k^{-\frac{d}{p}} f\left(\frac{x}{k}\right) .
$$

The transformation formula yields that $V_{k}$ is an isometry, whose inverse is given by

$$
\left(V_{k}^{-1} f\right)(x)=k^{\frac{d}{p}} f(k x) .
$$


For $f \in W^{2, p}\left(\Omega_{k}\right)$ we get by the chain rule $V_{k} f \in W^{2, p}(\Omega)$. Now consider $f \in W_{0}^{1, p}\left(\Omega_{k}\right) \cap\left\{f \in L^{p}\left(\Omega_{k}\right): \mathcal{L} f \in L^{p}\left(\Omega_{k}\right)\right\}$. Then there is a sequence $\left(f_{n}\right) \subseteq C_{c}^{\infty}\left(\Omega_{k}\right)$ which converges to $f$ in $W^{1, p}\left(\Omega_{k}\right)$. So $V_{k} f_{n} \in C_{c}^{\infty}(\Omega)$ with $\operatorname{supp}\left(V_{k} f_{n}\right)=k \operatorname{supp}\left(f_{n}\right)$ for all $k, n \in \mathbb{N}$. This sequence converges to $V_{k} f$, hence $V_{k} f \in W_{0}^{1, p}(\Omega)$. Moreover, we get

$$
\left(\mathcal{L} V_{k} f\right)(x)=k^{-\frac{d}{p}} B \frac{x}{k} \nabla f\left(\frac{x}{k}\right),
$$

hence $V_{k}^{-1} \mathcal{L} V_{k} f=\mathcal{L} f$, so $\mathcal{L} V_{k} f \in L^{p}(\Omega)$. For $V_{k}^{-1}$ one can argue analogously, so $V_{k}$ induces a bijection from $D\left(A_{k}\right)$ to $D\left(A_{1}\right)$. Now, for $f \in D\left(A_{k}\right)$ we derive

$$
\begin{aligned}
\left(V_{k}^{-1} A_{1} V_{k} f\right)(x) & =k^{-\frac{d}{p}} V_{k}^{-1} A_{1}\left[f\left(\frac{\dot{c}}{k}\right)\right](x) \\
& =k^{-\frac{d}{p}}\left[V_{k}^{-1}\left(\frac{1}{k^{2}} \sum_{i, j=1}^{d} q_{i j} D_{i} D_{j} f(\dot{\bar{k}})+\mathcal{L} f(\dot{\bar{k}})\right)\right](x) \\
& =\left(A_{k} f\right)(x),
\end{aligned}
$$

hence $\left(\lambda-A_{k}\right) f=V_{k}^{-1}\left(\lambda-A_{1}\right) V_{k} f$ for every $\lambda \in \mathbb{C}$ with $\operatorname{Re}(\lambda)>-\frac{\operatorname{tr}(B)}{p}$. This implies $R\left(\lambda, A_{k}\right)=V_{k}^{-1} R\left(\lambda, A_{1}\right) V_{k}$. Since $V_{k}: L^{p}\left(\Omega_{k}\right) \rightarrow L^{p}(\Omega)$ and $V_{k}^{-1}: L^{p}(\Omega) \rightarrow L^{p}\left(\Omega_{k}\right)$ are isometries, this concludes the proof.

Having this equality in hand, we shall finally show that the resolvents of $A_{\Omega, p}$ obey the inequality $\left\|R\left(\lambda, L_{\mathbb{R}^{d}, p}\right)\right\|_{\mathcal{L}\left(L_{p}\left(\mathbb{R}^{d}\right)\right)} \leq\left\|R\left(\lambda, A_{\Omega, p}\right)\right\|_{\mathcal{L}\left(L_{p}(\Omega)\right)}$ for $\operatorname{Re}(\lambda)>$ $-\frac{\operatorname{tr}(B)}{p}$. Since $\sigma\left(L_{\mathbb{R}^{d}, p}\right) \subseteq-\operatorname{tr}(B) / p+i \mathbb{R}$ by Proposition 5.1, we can approximate every $\mu \in \sigma\left(L_{\mathbb{R}^{d}, p}\right)$ by $\lambda \in \varrho\left(A_{\Omega, p}\right)$. The above inequality then implies the divergence of $\left\|R\left(\lambda, A_{\Omega, p}\right)\right\|_{\mathcal{L}\left(L^{p}(\Omega)\right)}$ for $\lambda \rightarrow \mu$. This yields $\sigma\left(L_{\mathbb{R}^{d}, p}\right) \subseteq \sigma\left(A_{\Omega, p}\right)$ and

$$
-\frac{\operatorname{tr}(B)}{p} \leq s\left(A_{\Omega, p}\right) \leq \omega_{0}\left(T_{\Omega, p}\right) \leq-\frac{\operatorname{tr}(B)}{p},
$$

as stated in Theorem 5.2.

Proposition 5.6. For $\operatorname{Re}(\lambda)>-\frac{\operatorname{tr}(B)}{p}$ we have

$$
\left\|R\left(\lambda, L_{\mathbb{R}^{d}, p}\right)\right\|_{\mathcal{L}\left(L^{p}\left(\mathbb{R}^{d}\right)\right)} \leq\left\|R\left(\lambda, A_{\Omega, p}\right)\right\|_{\mathcal{L}\left(L^{p}(\Omega)\right)} .
$$

Proof. Let $g \in C_{c}^{\infty}\left(\mathbb{R}^{d} \backslash\{0\}\right)$. Then $g \in D\left(A_{k}\right)$ for $k$ large enough. It follows

$$
\left\|A_{k} g-\mathcal{L} g\right\|_{L^{p}\left(\Omega_{k}\right)}=\left\|\frac{1}{k^{2}} \sum_{i, j=1}^{d} q_{i j} D_{i} D_{j} g\right\|_{L^{p}\left(\Omega_{k}\right)} \longrightarrow 0
$$


for $k \rightarrow \infty$. Now we consider $f=(\lambda-\mathcal{L}) g$. For $k$ large enough we have again $f \in D\left(A_{k}\right)$ and for $n \geq k$ we get with the previous lemma

$$
\begin{aligned}
& \left\|R\left(\lambda, A_{n}\right) f-R\left(\lambda, L_{\mathbb{R}^{d}, p}\right) f\right\|_{L^{p}\left(\mathbb{R}^{d}\right)}=\left\|R\left(\lambda, A_{n}\right)(\lambda-\mathcal{L}) g-g\right\|_{L^{p}\left(\Omega_{n}\right)} \\
= & \left\|R\left(\lambda, A_{n}\right)\left(\left(\lambda-A_{n}\right) g-\left(\lambda-A_{n}\right) g+(\lambda-\mathcal{L}) g\right)-g\right\|_{L^{p}\left(\Omega_{n}\right)} \\
= & \left\|R\left(\lambda, A_{n}\right)\left(A_{n}-\mathcal{L}\right) g\right\|_{L^{p}\left(\Omega_{n}\right)} \leq\left\|R\left(\lambda, A_{\Omega, p}\right)\right\|_{\mathcal{L}\left(L^{p}(\Omega)\right)}\left\|\left(A_{n}-\mathcal{L}\right) g\right\|_{L^{p}\left(\Omega_{n}\right)} \longrightarrow 0
\end{aligned}
$$

for $n \rightarrow \infty$. Note that the functions in the above calculation have to be restricted or extended by 0 to elements of the proper spaces. Now for each $\varepsilon>0$ and $f \in$ $\left(\lambda-L_{\mathbb{R}^{d}, p}\right) C_{c}^{\infty}\left(\mathbb{R}^{d} \backslash\{0\}\right)$ there exists a $k \in \mathbb{N}$ with

$$
\begin{aligned}
\left\|R\left(\lambda, L_{\mathbb{R}^{d}, p}\right) f\right\|_{L^{p}\left(\mathbb{R}^{d}\right)} & \leq\left\|R\left(\lambda, A_{k}\right) f\right\|_{L^{p}\left(\Omega_{k}\right)}+\varepsilon \\
& \leq\left\|R\left(\lambda, A_{\Omega, p}\right)\right\|_{\mathcal{L}\left(L^{p}(\Omega)\right)}\|f\|_{L^{p}\left(\mathbb{R}^{d}\right)}+\varepsilon .
\end{aligned}
$$

Thus we have

$$
\left\|R\left(\lambda, L_{\mathbb{R}^{d}, p}\right) f\right\|_{L^{p}\left(\mathbb{R}^{d}\right)} \leq\left\|R\left(\lambda, A_{\Omega, p}\right)\right\|_{\mathcal{L}\left(L^{p}(\Omega)\right)}\|f\|_{L^{p}\left(\mathbb{R}^{d}\right)}
$$

for all $f \in\left(\lambda-L_{\mathbb{R}^{d}, p}\right) C_{c}^{\infty}\left(\mathbb{R}^{d} \backslash\{0\}\right)$. Since $C_{c}^{\infty}\left(\mathbb{R}^{d} \backslash\{0\}\right)$ is a core for $L_{\mathbb{R}^{d}, p}$ by Lemma 5.4, the assertion follows.

\section{References}

[1] R. A. AdAms, "Sobolev Spaces", Academic Press, New York, 1978.

[2] W. ARENDT and P. BÉNILAN, Wiener regularity and heat semigroups on spaces of continuous functions, In: "Topics in Nonlinear Analysis", Vol. 35, Progr. Nonlinear Differential Equations Appl., Birkhäuser, Basel, 1999, 29-49.

[3] M. BERTOLDI and L. LORENZI, Analytic methods for markov semigroups, preprint.

[4] G. DA PRATO and A. LunARDI, Elliptic operators with unbounded drift coefficients and Neumann boundary condition, J. Differential Equations 198 (2004), 35-52.

[5] R. Dautray and J.-L. Lions, "Mathematical Analysis and Numerical Methods for Science and Technology", Vol. 1, Springer-Verlag, Berlin, 1990.

[6] E.B. DAVIES and B. Simon, $L^{1}$-properties of intrinsic Schrödinger semigroups, J. Funct. Anal. 65 (1986), 126-146.

[7] K.-J. Engel and R. NAgel, "One-Parameter Semigroups for Linear Evolution Equations", Vol. 194, Graduate Texts in Mathematics, Springer-Verlag, New York, 2000.

[8] S. Fornaro, G. Metafune and E. Priola, Gradient estimates for Dirichlet parabolic problems in unbounded domains, J. Differential Equations 205 (2004), 329-353.

[9] M. Geissert, H. HeCK and M. Hieber, $L^{p}$-theory of the Navier-Stokes flow in the exterior of a moving or rotating obstacle, 2005, preprint.

[10] M. GeIsSeRT, H. Heck, M. HiebeR and I. Wood, The Ornstein-Uhlenbeck semigroup in exterior domains, Arch. Math. (Basel), to appear.

[11] D. Gilbarg and N. S. Trudinger, "Elliptic Partial Differential Operators of Second Order. Second Edition", Vol. 224, A Series of Comprehensive Studies in Mathematics, Springer-Verlag, Berlin, 1983.

[12] M. HIEBER and J. PRÜSS, Functional calculi for linear operators in vector-valued $L^{p}$ spaces via the transference principle, Adv. Differential Equations 3 (1998), 847-872. 
[13] M. HIEBER - O. SAWADA, The Navier-Stokes equations in $\mathbb{R}^{n}$ with linearly growing initial data, Arch. Ration. Mech. Anal. 175 (2005), 269-285.

[14] T. HishidA, An existence theorem for the Navier-Stokes flow in the exterior of a rotating obstacle, Arch. Ration. Mech. Anal. 150 (1999), 307-348.

[15] G. Metafune, $L^{p}$-spectrum of Ornstein-Uhlenbeck operators, Ann. Scuola Norm. Sup. Pisa Cl. Sci. (4) 30 (2001), 97-124.

[16] G. Metafune, D. Pallara and E. Priola, Spectrum of Ornstein-Uhlenbeck operators in $L^{p}$ spaces with respect to invariant measures, J. Funct. Anal. 196 (2002), 40-60.

[17] G. Metafune, D. Pallara and V. Vespri, $L^{p}$-estimates for a class of elliptic operators with unbounded coefficients in $\mathbb{R}^{n}$, Houston Math. J. 31 (2005), 605-620.

[18] G. Metafune, J. Prüss, A. Rhandi and R. Schnaubelt, The domain of the OrnsteinUhlenbeck operator on an $L^{p}$-space with invariant measure, Ann. Scuola Norm. Sup. Pisa Cl. Sci. (5) 1 (2002), 471-485.

[19] H. H. SCHAEFER, "Banach Lattices and Positive Operators", Vol. 215 of Die Grundlehren der mathematischen Wissenschaften, Springer-Verlag, New York, 1974.

Technische Universität Darmstadt Fachbereich Mathematik

Schlossgartenstr. 7

D-64289 Darmstadt, Germany

haller@mathematik.tu-darmstadt.de

Technische Universität Darmstadt

Fachbereich Mathematik

Schlossgartenstr. 7

D-64289 Darmstadt, Germany

wiedl@mathematik.tu-darmstadt.de 